\title{
UN EJEMPLO DE BUENA PRÁCTICA DE EVALUACIÓN FORMATIVA Y COMPARTIDA EN EL TERCER CURSO DEL GRADO DE EDUCACIÓN INFANTIL
}

An example of good practice of formative and shared assessment in the third year of the Pre-service Early Childhood Teacher Education

Um exemplo de boas práticas de avaliação formativa e partilhada no terceiro ano da licenciatura em educação pré-escolar

\author{
Miriam Molina Soria (1) \\ Cristina Pascual Arias (2)
}

Víctor Manuel López Pastor (3)

(1) Universidad de Valladolid, España. Teléfono: +34 921112321. Correo electrónico: miriam.molina@uva.es

(2) Universidad de Valladolid, España. Teléfono: +34 921112321. Correo electrónico: cristina.pascual@uva.es

(3) Universidad de Valladolid, España. Teléfono: +34 921112321. Correo electrónico: vlopez@mpc.uva.es

\section{Resumen}

Este trabajo presenta una experiencia de buena práctica de evaluación formativa y compartida (EFyC) en el tercer curso del grado de Educación Infantil. Se trata de un Proyecto de Aprendizaje Tutorado (PAT) utilizado en la Formación Inicial del Profesorado (FIP), durante el segundo cuatrimestre del curso 2018/19, en la asignatura de Fundamentos y Didáctica de la Educación Corporal Infantil. Los resultados han sido positivos para el alumnado, gracias a la metodología de aprendizaje y al sistema de EFyC llevada a cabo. Se obtienen valores muy altos en rendimiento y éxito académico. La carga de trabajo del alumnado está dentro de las horas oficialmente previstas de trabajo no presencial, por lo que debe ser considerada adecuada.

Palabras clave: Proyectos de aprendizaje tutorado, formación inicial del profesorado; evaluación formativa; evaluación compartida 


\begin{abstract}
This work presents a good practice experience of Formative and Shared Assessment (F\&SA) in the third year of the degree in Pre-service Early Childhood Teacher Education. It is a Tutored Learning Project (TLP) used in Pre-service Teacher Education (PTE), during the second semester of the 2018/19 school year, in the subject of Physical Education in Childhood Education. The results have been positive for the students, thanks to the learning methodology and the F\&SA system carried out. Very high values are obtained in performance and academic success. The workload of the students is within the officially foreseen hours of non-contact work, so it cannot be considered as excessive.
\end{abstract}

Keywords: Tutored learning projects, pre-service teacher education; formative assessment; shared assessment

\title{
Resumo
}

Este trabalho apresenta uma experiência de boas práticas em avaliação formativa e partilhada (AFyP), no terceiro ano da licenciatura da Educação Pré-escolar. Trata-se de um Projeto de Aprendizagem Tutorado (PAT), utilizado na Formação Inicial de Professores (FIP), durante o segundo quadrimestre do ano letivo 2018/19, na disciplina Fundamentos e Didática da Educação Corporal Pré-escolar. Os resultados foram positivos para os alunos, graças à metodologia de aprendizagem e ao sistema AFyP usado. Foram obtidos valores muito altos ao nível do desempenho e sucesso académico. A carga de trabalho dos alunos está dentro das horas oficialmente previstas de trabalho não presencial e portanto, pode ser considerada adequada.

Palavras-chave: Projetos de aprendizagem tutorados, formação inicial de professores; avaliação formativa; avaliação partilhada

\section{Introducción}

La evaluación formativa ayuda a mejorar los procesos de enseñanza-aprendizaje y nos ayuda a obtener información que permite saber cómo ayudar al alumnado a mejorar y a aprender más (López-Pastor, 2009; López-Pastor y Pérez-Pueyo, 2017). En este trabajo vamos a presentar un sistema de evaluación formativa y compartida (EFyC) utilizado en una asignatura de formación inicial del profesorado (FIP) de educación infantil. El 
proceso de EFyC se da de forma continua durante toda la asignatura, en cada documento que entrega el alumnado sobre las diferentes actividades de aprendizaje que se llevan a cabo durante el cuatrimestre. La asignatura finaliza con un proceso de autoevaluación y evaluación compartida. La utilización de estos sistemas de EFyC demuestra ser un proceso clave para lograr mejores aprendizajes y conseguir una buena calidad de los trabajos presentados.

\section{Contextualización}

Esta experiencia se lleva a cabo en la Facultad de Educación de Segovia (Universidad de Valladolid), en $3^{\circ}$ de grado Educación Infantil, en la asignatura de Fundamentos y Didáctica de la Educación Corporal Infantil, durante el segundo cuatrimestre del curso 2018/19. Esta asignatura consta de 6 créditos: 60 horas lectivas y 90 horas de trabajo no presencial, con 38 alumnas.

\section{Diseño y desarrollo}

En la tabla 1 se presentan las diferentes actividades de aprendizaje de la asignatura, con los procesos de evaluación de cada una y el porcentaje de la calificación final.

Tabla 1.

Resumen de actividades de aprendizaje, evaluación y peso en la calificación final.

\begin{tabular}{lllc}
\hline Actividad de aprendizaje & Instrumento evaluación & Evaluación formativa & $\begin{array}{l}\text { Peso en } \\
\text { calificación }\end{array}$ \\
\hline 1-PATo & $\begin{array}{l}\text { Escala descriptiva } \\
\text { (rúbrica) }\end{array}$ & $\begin{array}{l}\text { En todos los casos cada } \\
\text { documento entregado se } \\
\text { revisa por el profesorado, } \\
\text { aportando feed-back para } \\
\text { su mejora }\end{array}$ & 30 \\
$\begin{array}{l}\text { 2-Fichas de sesiones } \\
\text { prácticas }\end{array}$ & $\begin{array}{l}\text { Escala descriptiva } \\
\text { (rúbrica) }\end{array}$ & $\begin{array}{l}\text { Si hay cosas que tengan } \\
\text { que ser corregidas, se da } \\
\text { 3-Examen parcial con } \\
\text { coevaluación inmediata }\end{array}$ & Planilla \\
$\begin{array}{l}\text { 4-Mapas conceptuales y } \\
\text { dossier asignatura }\end{array}$ & $\begin{array}{l}\text { Escala descriptiva } \\
\text { (rúbrica) }\end{array}$ & 20 & 20 \\
$\begin{array}{l}\text { 5-Lecturas y tertulias } \\
\text { dialógicas }\end{array}$ & $\begin{array}{l}\text { Escala descriptiva } \\
\text { (rúbrica) }\end{array}$ & & 10 \\
\hline
\end{tabular}

\section{Evaluación}

La evaluación de la asignatura se lleva a cabo a través de un cuestionario anónimo con escala tipo Likert (1-5) y dos escalas de autopercepción de competencia del alumnado con escala tipo Likert (0-4), dividido en tres escalas: "competencias transversales", “competencias docentes generales" y "competencias docentes específicas de EF". 
Actualmente sólo disponemos de los datos de la escala pretest de autopercepción de competencia, que presentamos en los anexos I, II y III.

Como puede comprobarse en el Anexo I, la autopercepción de competencias transversales del alumnado es bastante elevada en la mayoría de los ítems. Las medias más altas se dan en los ítems de "desarrollar habilidades en las relaciones interpersonales" (3.73), "comunicarse de forma oral y escrita" y "trabajar en equipo" (3.45). Los ítems con medias más bajas son "conocer una lengua extranjera" (2.55) y "analizar y sintetizar" (2.95).

En el Anexo II puede comprobarse que la autopercepción de competencias docentes generales es también bastante alta, aunque ligeramente menor que en la tabla anterior. El ítem que tiene la media aritmética más alta es "implicar al alumnado en su aprendizaje" (3.32), seguido de tres ítems $(2.3,2.4,2.12)$ con 3.00. Los ítems que tienen una media aritmética más baja son "participar en la gestión del centro" (2.41) y "poner en marcha procesos de investigación en el aula" (2.45).

En el Anexo III puede comprobarse que la autopercepción de competencias docentes específicas de EF tiene los valores más bajos de las tres, con la mayoría de los ítems por debajo del 3. Los ítems con un valor más alto son: "saber utilizar el juego como recurso didáctico y como contenido de enseñanza" (3.27) y "diseñar en un plan de hábitos de vida saludable y de práctica de actividad física regular" (2.91). El valor más bajo (2.23) se repite en tres ítems $(3.2,3.3,3.12)$.

En junio podremos comparar y analizar estos datos con los que se obtengan en la escala postest.

\section{Conclusiones}

Como puede comprobarse, la autopercepción de competencias del alumnado es bastante alta en general (2.23-3.73), aunque el bloque donde se sienten menos competentes es el que hace referencia a las competencias docentes específicas de EF. Son unos resultados lógicos, dado que es la primera asignatura que cursan sobre EF en la carrera.

En estos momentos no disponemos de los datos de carga de trabajo ni rendimiento, ni del cuestionario de evaluación de la asignatura, dado que esos datos se recogen al finalizar en junio. Estos resultados serán presentados en el congreso. Hasta el momento la asignatura está teniendo un proceso correcto, similar a cursos anteriores, por lo que esperamos que los resultados sean también positivos.

Un ejemplo de buena práctica de evaluación formativa y compartida en el tercer curso del grado de 


\section{Referencias}

López-Pastor, V. M. (coord.) (2009). Evaluación Formativa y Compartida en Educación Superior. Narcea: Madrid.

López-Pastor, V. M., \& Pérez-Pueyo, A. (Coords.) (2017). Evaluación formativa y compartida en educación: experiencias de éxito en todas las etapas educativas. León: Universidad de León. (e-book). Recuperado de https://buleria.unileon.es/handle/10612/5999 


\section{Anexos}

Anexo I. Tabla 2.

Datos del cuestionario de autopercepción de competencias.

\begin{tabular}{lc}
\hline \multicolumn{1}{c}{ Bloque 1: competencias transversales } & $\begin{array}{c}\text { Media } \\
\text { aritmética }\end{array}$ \\
& 2,95 \\
11.- Analizar y sintetizar & 3,14 \\
12.- Organizar y planificar & 3,45 \\
13.- Comunicarse de forma oral y escrita & 3,09 \\
14.- Comunicarse gestual y corporalmente & 2,55 \\
15.- Conocer una lengua extranjera & 3,18 \\
16.- Utilizar las Tecnologías de la Información y la Comunicación en el ámbito de estudio & 3,45 \\
17.- Trabajar en equipo & 3,73 \\
18.- Desarrollar habilidades en las relaciones interpersonales (empatía, asertividad, respeto & \\
y escucha) & 3,00 \\
19.- Desarrollar habilidades intrapersonales (autoestima, motivación y confianza en un \\
mismo) & 3,09 \\
110.- Razonar de forma crítica y reflexiva & 3,14 \\
111.- Desarrollar un compromiso ético & 3,32 \\
112.- Desarrollar un aprendizaje autónomo & 3,14 \\
113.- Adaptarse a situaciones nuevas & 3,00 \\
114.- Desarrollar la creatividad &
\end{tabular}

Anexo II. Tabla 3

Datos del cuestionario de autopercepción de competencias.

\begin{tabular}{lc}
\hline \multicolumn{1}{c}{ Bloque 2: Competencias docentes } & Media \\
& aritmética \\
21.- Conocer las características organizativas de os centros educativos & 2,86 \\
22.- Elaborar propuestas de cambio de la realidad educativa & 2,59 \\
23.- Diseñar situaciones de aprendizaje & 3,00 \\
24.- Animar situaciones de aprendizaje & 3,00 \\
25.- Gestionar la progresión de los aprendizajes & 2,64 \\
26.- Diseñar estrategias de atención a la diversidad & 2,64 \\
27.- Poner en práctica estrategias de atención a la diversidad & 2,64 \\
28.- Implicar al alumnado en su aprendizaje & 3,32 \\
29.- Implicar al alumnado en la vida del centro & 2,82 \\
210.- Participar en la gestión del Centro & 2,41 \\
211.- Informar a las familias sobre la evolución de su hijo/a & 2,82 \\
212.- Implicar a las familias en el aprendizaje de su hijo/a & 3,00 \\
213.- Implicar a las familias en la vida del centro & 2,77 \\
214.- Afrontar los deberes y dilemas éticos de la profesión & 2,77 \\
215.- Atender al propio desarrollo profesional docente (autoevaluación, lecturas, cursos, \\
jornadas, congresos, redes de trabajo) & 2,95 \\
216.- Desarrollar procesos de innovación educativa en el aula & 2,86 \\
217.- Poner en marcha procesos de investigación en el aula & 2,45 \\
\hline
\end{tabular}

Un ejemplo de buena práctica de evaluación formativa y compartida en el tercer curso del grado de Educación Infantil 
Anexo III. Tabla 4.

Datos del cuestionario de autopercepción de competencias.

Bloque 3: competencias específicas docentes de educación física

31.- Diseñar, aplicar y analizar intervenciones didácticas en el área de Educación Física

32.- Diseñar, desarrollar y evaluar los procesos de enseñanza-aprendizaje relativos a la actividad física y el deporte con atención a las Necesidades Específicas de Apoyo Educativo

33.- Conocer y promover las diferentes manifestaciones motrices que forman parte de la cultura tradicional

34.- Conocer y aplicar los fundamentos biológicos, fisiológicos, de maduración evolutiva y el desarrollo psicomotor

35.- Diseñar, modificar y/o adaptar al contexto educativo situaciones motrices orientadas al desarrollo y perfeccionamiento de las habilidades motrices

36.- Conocer elementos y fundamentos de la expresión corporal y la comunicación no verbal y su valor formativo y cultural

37.- Saber utilizar el juego como recurso didáctico y como contenido de enseñanza

38.- Conocer los fundamentos básicos de la iniciación deportiva escolar y diseñar tareas específicas para utilizarlos en el ámbito de la enseñanza

39.- Identificar los riesgos que se derivan para la salud de la práctica de actividades físicas inadecuadas

310.- Diseñar en un plan de hábitos de vida saludable (higiene y alimentación) y de práctica de- actividad física regular

311.- Saber aplicar los fundamentos (técnicas) de las actividades físicas en el medio natural

312.- Saber utilizar diferentes instrumentos de evaluación en el área de Educación Física

3.13.- Tener capacidad de reflexión sobre el proceso de enseñanza/aprendizaje y las distintas metodologías dentro de las clases de Educación Física

314.- Analizar y comunicar, de manera crítica y fundamentada, el valor de la actividad física y el deporte y sus posibilidades de contribuir al desarrollo y bienestar de las personas
Media

aritmética

2,36

2,23

2,23

2,32

2,23

Un ejemplo de buena práctica de evaluación formativa y compartida en el tercer curso del grado de Educación Infantil 